University of Nebraska - Lincoln

DigitalCommons@University of Nebraska - Lincoln

$11-9-2006$

\title{
Nondipole Effects in Double Photoionization of He
}

\author{
A.Y. Istomin \\ University of Nebraska - Lincoln \\ N. L. Manakov \\ Voronezh State University, manakov@phys.vsu.ru
}

A. V. Meremianin

Voronezh State University, meremianin@phys.vsu.ru

Anthony F. Starace

University of Nebraska-Lincoln, astarace1@unl.edu

Follow this and additional works at: https://digitalcommons.unl.edu/physicsstarace

Part of the Physics Commons

Istomin, A.Y.; Manakov, N. L.; Meremianin, A. V.; and Starace, Anthony F., "Nondipole Effects in Double Photoionization of He" (2006). Anthony F. Starace Publications. 90.

https://digitalcommons.unl.edu/physicsstarace/90

This Article is brought to you for free and open access by the Research Papers in Physics and Astronomy at DigitalCommons@University of Nebraska - Lincoln. It has been accepted for inclusion in Anthony F. Starace Publications by an authorized administrator of DigitalCommons@University of Nebraska - Lincoln. 


\title{
Nondipole Effects in Double Photoionization of $\mathrm{He}$
}

\author{
A. Y. Istomin*, N. L. Manakov ${ }^{\dagger}$, A. V. Meremianin ${ }^{\dagger}$ and A. F. Starace* \\ ${ }^{*}$ Department of Physics and Astronomy, The University of Nebraska, Lincoln, NE 68588-0111 \\ ${ }^{\dagger}$ Physics Department, Voronezh State University, Voronezh 394006, Russia
}

\begin{abstract}
Lowest-order nondipole effects are studied in double photoionization (DPI) of the He atom. $A b$ initio parametrizations of the quadrupole transition amplitude for DPI from the ${ }^{1} S_{0^{-}}$ state are presented in terms of the exact two-electron reduced matrix elements. Parametrizations for the dipole-quadrupole triply differential cross section (TDCS) and doubly differential cross section (DDCS) are presented in terms of polarization-independent amplitudes for the case of an elliptically polarized photon. Expressions for the DDCS in terms of the reduced two-electron matrix elements are also given. A general analysis of retardation-induced asymmetries of the TDCS including the circular dichroism effect at equal energy sharing is presented. Our numerical results exhibit a nondipole forward-backward asymmetry in the TDCS for DPI of He at an excess energy of $450 \mathrm{eV}$ that is in qualitative agreement with existing experimental data.
\end{abstract}

Keywords: double ionization, nondipole effects, circular dichroism, helium, quadrupole amplitude PACS: $32.80 . \mathrm{Fb}$

\section{GENERAL RESULTS FOR THE DPI QUADRUPOLE TRANSITION AMPLITUDE}

We consider double photoionization (DPI) from the ${ }^{1} S_{0}$ state $|0\rangle$ to the final two-electron singlet state $\left\langle\mathbf{p}_{1} \mathbf{p}_{2}\right\rangle$, with asymptotic electron momenta $\mathbf{p}_{1}$ and $\mathbf{p}_{2}$, in the nonrelativistic domain of photon energies taking into account lowest-order retardation corrections. Because neither orbital nor spin-dependent parts of the magnetic dipole interaction contribute to the transition amplitude $A$ for nonrelativistic photon energies [1], the spin dependence of the two-electron wave functions is suppressed in our analysis. The dipolequadrupole TDCS for DPI is:

$$
\frac{d^{3} \sigma}{d \varepsilon_{1} d \Omega_{1} d \Omega_{2}} \equiv \sigma=\mathscr{A}|A|^{2},
$$

where $\mathscr{A}=4 \pi^{2} \alpha p_{1} p_{2} / \omega$ is a normalization factor, and $\alpha=1 / 137.036$. Atomic units are used throughout this paper. The amplitude $A$ involving E1 and E2 components has the form (where $\mathbf{e}$ is the photon polarization vector and $\mathbf{k}$ is the photon wavevector),

$$
A=A_{d}+A_{q}=\left\langle\mathbf{p}_{1} \mathbf{p}_{2}\left|(\mathbf{e} \cdot \mathbf{D})+\left(\{\hat{\mathbf{k}} \otimes \mathbf{e}\}_{2} \cdot Q_{2}\right)\right| 0\right\rangle,
$$

for both velocity $(\mathrm{V})$ and length $(\mathrm{L})$ gauges of the electron-photon interaction. $\{\mathbf{a} \otimes \mathbf{b}\}_{2 m}$ is the irreducible tensor product of rank 2. In the V-gauge, $\mathbf{D} \equiv \mathbf{D}^{(V)}=-i\left(\nabla_{1}+\nabla_{2}\right)$ and $Q_{2 m} \equiv Q_{2 m}^{(V)}=\alpha \omega\left(\left\{\mathbf{r}_{1} \otimes \nabla_{1}\right\}_{2 m}+\left\{\mathbf{r}_{2} \otimes \nabla_{2}\right\}_{2 m}\right)$. In the L-gauge, $\mathbf{D} \equiv \mathbf{D}^{(L)}=i \omega\left(\mathbf{r}_{1}+\mathbf{r}_{2}\right)$ and $Q_{2 m} \equiv Q_{2 m}^{(L)}=-(1 / 2) \alpha \omega^{2}\left(\left\{\mathbf{r}_{1} \otimes \mathbf{r}_{1}\right\}_{2 m}+\left\{\mathbf{r}_{2} \otimes \mathbf{r}_{2}\right\}_{2 m}\right)$. (See Ref. [2] for details.) 
In the electric dipole approximation (EDA), the parametrization of the EDA amplitude $A_{d}$ in terms of scalar products of the vectors $\mathbf{e}, \hat{\mathbf{p}}_{1}$, and $\hat{\mathbf{p}}_{2}$ is well-known $[3,4]$ :

$$
A_{d}=f_{1}\left(\mathbf{e} \cdot \hat{\mathbf{p}}_{1}\right)+f_{2}\left(\mathbf{e} \cdot \hat{\mathbf{p}}_{2}\right) .
$$

In this equation, $f_{1} \equiv f\left(p_{1}, p_{2}, \cos \theta\right)$ and $f_{2} \equiv f\left(p_{2}, p_{1}, \cos \theta\right)$ [where $\theta_{12} \equiv \theta$ is the mutual ejection angle, $\left.\cos \theta=\left(\hat{\mathbf{p}}_{1} \cdot \hat{\mathbf{p}}_{2}\right)\right]$ are defined by a single function,

$$
f\left(p, p^{\prime}, \cos \theta\right)=\sum_{l=1}^{\infty}(-1)^{l+1}\left[\sum_{l^{\prime}=l \pm 1} D_{l l^{\prime}}\left(p, p^{\prime}\right)\right] P_{l}^{(1)}(\cos \theta),
$$

where $P_{l}^{(n)}(x)$ is the $n$-th derivative of the Legendre polynomial $P_{l}(x), P_{l}^{(n)}(x)=$ $\left(d^{n} / d x^{n}\right) P_{l}(x)$. The energy-dependent coefficient $D_{l l^{\prime}}\left(p, p^{\prime}\right)$ is given by $D_{l l^{\prime}}\left(p, p^{\prime}\right)=$ $\left[(2 l+1)\left(2 l^{\prime}+1\right) \max \left(l, l^{\prime}\right)\right]^{-1 / 2} d_{l l^{\prime}}\left(p, p^{\prime}\right)$, where $d_{l l^{\prime}}\left(p, p^{\prime}\right) \equiv\left\langle p p^{\prime} ;\left(l l^{\prime}\right) 1|| \mathbf{D}|| 0\right\rangle$ is the reduced matrix element of the operator $D$ between the ${ }^{1} S_{0}$-state and the $P$-wave component of the final state $\left|\mathbf{p p}^{\prime}\right\rangle$, with photoelectron angular momenta $l$ and $l^{\prime}=l \pm 1$. We use an expansion of $\left|\mathbf{p} \mathbf{p}^{\prime}\right\rangle$ in terms of modified bipolar harmonics $\mathscr{C}_{L M}^{l l^{\prime}}\left(\hat{\mathbf{p}}, \hat{\mathbf{p}}^{\prime}\right)$,

$$
\left|\mathbf{p p}^{\prime}\right\rangle=\sum_{l l^{\prime} L M} \mathscr{C}_{L M}^{l^{\prime}{ }^{*}}\left(\hat{\mathbf{p}}, \hat{\mathbf{p}}^{\prime}\right)\left|p p^{\prime} ;\left(l l^{\prime}\right) L M\right\rangle
$$

Similarly to the derivations of Eqs. (3) and (4) in Ref. [3], to derive a parametrization of the quadrupole amplitude $A_{q}$ we use the expansion (5) and the reduction formulae [3] for the rank-2 bipolar harmonics. Thus, $A_{q}$ may be presented as follows [2, 5]:

$$
A_{q}=g_{1}\left(\mathbf{e} \cdot \hat{\mathbf{p}}_{1}\right)\left(\hat{\mathbf{p}}_{1} \cdot \hat{\mathbf{k}}\right)+g_{2}\left(\mathbf{e} \cdot \hat{\mathbf{p}}_{2}\right)\left(\hat{\mathbf{p}}_{2} \cdot \hat{\mathbf{k}}\right)+g_{s}\left[\left(\mathbf{e} \cdot \hat{\mathbf{p}}_{1}\right)\left(\hat{\mathbf{p}}_{2} \cdot \hat{\mathbf{k}}\right)+\left(\mathbf{e} \cdot \hat{\mathbf{p}}_{2}\right)\left(\hat{\mathbf{p}}_{1} \cdot \hat{\mathbf{k}}\right)\right],
$$

where the generally complex parameters $g_{1,2}$ and $g_{s}$ depend on $p_{1}, p_{2}$, and $\theta$. The parameter $g_{s}$ is symmetric in $p_{1}$ and $p_{2}, g_{s} \equiv g_{s}\left(p_{1}, p_{2}, \cos \theta\right)=g_{s}\left(p_{2}, p_{1}, \cos \theta\right)$, while $g_{1}$ and $g_{2}$ are expressed in terms of a single function, $g\left(p, p^{\prime}, \cos \theta\right)$, with $g_{1} \equiv g\left(p_{1}, p_{2}, \cos \theta\right)$ and $g_{2} \equiv g\left(p_{2}, p_{1}, \cos \theta\right)$. The explicit forms of the functions $g\left(p, p^{\prime}, \cos \theta\right)$ and $g_{s}\left(p, p^{\prime}, \cos \theta\right)$ are as follows:

$$
\begin{aligned}
g_{s}\left(p, p^{\prime}, \cos \theta\right)= & \sum_{l=1}^{\infty}(-1)^{l+1}\left[\sum_{l^{\prime}=l \pm 2} Q_{l l^{\prime}}\left(p, p^{\prime}\right) P_{\frac{l+l^{\prime}}{2}}^{(2)}(\cos \theta)\right. \\
& \left.+\sqrt{6} Q_{l l}\left(p, p^{\prime}\right)\left(P_{l+1}^{(2)}(\cos \theta)-\frac{2 l+3}{2} P_{l}^{(1)}(\cos \theta)\right)\right], \\
g\left(p, p^{\prime}, \cos \theta\right)= & \sum_{l=2}^{\infty}(-1)^{l}\left[\sum_{l^{\prime}=l \pm 2} Q_{l l^{\prime}}\left(p, p^{\prime}\right)+\sqrt{6} Q_{l l}\left(p, p^{\prime}\right)\right] P_{l}^{(2)}(\cos \theta),
\end{aligned}
$$

where $Q_{l l^{\prime}}\left(p, p^{\prime}\right)=\left[4\left(l+l^{\prime}-2\right) ! /\left(l+l^{\prime}+3\right) !\right]^{1 / 2} q_{l l^{\prime}}\left(p, p^{\prime}\right)$ and $q_{l l^{\prime}}\left(p, p^{\prime}\right) \equiv$ $\left\langle p p^{\prime} ;\left(l l^{\prime}\right) 2\left\|Q_{2}\right\| 0\right\rangle$ is the reduced matrix element of the operator $Q_{2 m}$ between the initial ${ }^{1} S_{0}$-state, $|0\rangle$, and the $D$-wave component of the two-electron continuum state $\left|\mathbf{p p}^{\prime}\right\rangle$ with photoelectron angular momenta $l$ and $l^{\prime}=l, l \pm 2$. 
An alternative parametrization of the DPI amplitude $A$ in terms of orthogonal vectors $\mathbf{p}_{+}=\left(\hat{\mathbf{p}}_{1}+\hat{\mathbf{p}}_{2}\right) / 2$ and $\mathbf{p}_{\sim}=\left(\hat{\mathbf{p}}_{1}-\hat{\mathbf{p}}_{2}\right) / 2$ and the symmetrized $\left(f^{(\mathbf{g})}=f_{1}+f_{2}\right.$ and $\left.g_{ \pm}^{(\mathrm{g})}=g_{1}+g_{2} \pm 2 g_{s}\right)$ and antisymmerized $\left(f^{(\mathrm{u})}=f_{1}-f_{2}\right.$ and $\left.g^{(\mathrm{u})}=g_{1}-g_{2}\right)$ amplitudes is rather obvious and can be easily obtained from Eqs. (3), (4), (6), and (7) [2]. The results in Eqs. (3), (4), (6) and (7) are general and do not depend upon the dynamical model used for calculation of the reduced matrix elements $d_{l l^{\prime}}\left(p, p^{\prime}\right)$ and $q_{l l^{\prime}}\left(p, p^{\prime}\right)$.

\section{PARAMETRIZATIONS AND NUMERICAL ESTIMATES FOR THE DIPOLE-QUADRUPOLE TDCS}

For the most general case of DPI by elliptically polarized light (described by the complex polarization vector $\left.\mathbf{e}\left[\left(\mathbf{e} \cdot \mathbf{e}^{*}\right)=1\right]\right)$, the TDCS in Eq. (1) (neglecting the small terms $\left.\sim\left|A_{q}\right|^{2}\right)$ has a model-independent parametrization similar to that for dipole DPI [3],

$$
\begin{aligned}
\sigma= & \mathscr{A}\left\{c_{1}\left|\mathbf{e} \cdot \hat{\mathbf{p}}_{1}\right|^{2}+c_{2}\left|\mathbf{e} \cdot \hat{\mathbf{p}}_{2}\right|^{2}+\operatorname{Re} c_{3}\left[(1-\ell)\left(\left(\hat{\mathbf{p}}_{1} \cdot \hat{\mathbf{p}}_{2}\right)-\left(\hat{\mathbf{k}} \cdot \hat{\mathbf{p}}_{1}\right)\left(\hat{\mathbf{k}} \cdot \hat{\mathbf{p}}_{2}\right)\right)\right.\right. \\
& \left.\left.+2 \ell\left(\hat{\boldsymbol{\varepsilon}} \cdot \hat{\mathbf{p}}_{1}\right)\left(\hat{\boldsymbol{\varepsilon}} \cdot \hat{\mathbf{p}}_{2}\right)\right]+\xi \operatorname{Im} c_{3}\left(\hat{\mathbf{k}} \cdot\left[\hat{\mathbf{p}}_{1} \times \hat{\mathbf{p}}_{2}\right]\right)\right\},
\end{aligned}
$$

where, in contrast to the dipole case, the coefficients $c_{i}$ depend upon $\mathbf{k}$ :

$$
\begin{aligned}
& c_{1}=\left|f_{1}\right|^{2}+2 \operatorname{Re}\left[f_{1} g_{1}^{*}\left(\hat{\mathbf{k}} \cdot \hat{\mathbf{p}}_{1}\right)+f_{1} g_{s}^{*}\left(\hat{\mathbf{k}} \cdot \hat{\mathbf{p}}_{2}\right)\right], \\
& c_{2}=\left|f_{2}\right|^{2}+2 \operatorname{Re}\left[f_{2} g_{2}^{*}\left(\hat{\mathbf{k}} \cdot \hat{\mathbf{p}}_{2}\right)+f_{2} g_{s}^{*}\left(\hat{\mathbf{k}} \cdot \hat{\mathbf{p}}_{1}\right)\right], \\
& c_{3}=f_{1} f_{2}^{*}+\left(f_{1} g_{s}^{*}+f_{2}^{*} g_{1}\right)\left(\hat{\mathbf{k}} \cdot \hat{\mathbf{p}}_{1}\right)+\left(f_{2}^{*} g_{s}+f_{1} g_{2}^{*}\right)\left(\hat{\mathbf{k}} \cdot \hat{\mathbf{p}}_{2}\right) .
\end{aligned}
$$

The parameters $\ell$ and $\xi$ in Eq. (8) are the degrees of linear and circular polarization of an elliptically polarized photon; $\ell=\mathbf{e}^{2}=\sqrt{1-\xi^{2}}, \xi \equiv i\left(\hat{\mathbf{k}} \cdot\left[\mathbf{e} \times \mathbf{e}^{*}\right]\right)$, and the unit vector $\hat{\boldsymbol{\varepsilon}}$ is directed along the major axis of the polarization ellipse. The photon polarization dependence of the dipole-quadrupole TDCS is thus determined by four real $\mathbf{k}$-dependent parameters, $c_{1}, c_{2}, \operatorname{Re} c_{3}$, and $\operatorname{Im} c_{3}$. These parameters may be determined from four measurements with different polarizations of the photon beam, e.g., two experiments with linearly polarized photons and two experiments with circularly polarized photons. For circularly polarized photons $(\ell=0, \xi= \pm 1)$ Eq. (8) simplifies,

$$
\begin{aligned}
\sigma=\frac{\mathscr{A}}{2}\left(c_{1}\left[\hat{\mathbf{k}} \times \hat{\mathbf{p}}_{1}\right]^{2}+c_{2}\left[\hat{\mathbf{k}} \times \hat{\mathbf{p}}_{2}\right]^{2}+\operatorname{Re} c_{3}\left(\left[\hat{\mathbf{k}} \times \hat{\mathbf{p}}_{1}\right] \cdot\left[\hat{\mathbf{k}} \times \hat{\mathbf{p}}_{2}\right]\right)\right. \\
\left.+2 \xi \operatorname{Im} c_{3}\left(\hat{\mathbf{k}} \cdot\left[\hat{\mathbf{p}}_{1} \times \hat{\mathbf{p}}_{2}\right]\right)\right) .
\end{aligned}
$$

The TDCSs in Eqs. (8) and (10) both contain a term that is proportional to $\xi$, which is responsible for the circular dichroism $(C D)$ effect. This effect is usually characterized by the absolute $\mathrm{CD}$ parameter, $\Delta_{c d} \equiv \sigma(\xi=+1)-\sigma(\xi=-1)$ :

$$
\Delta_{c d}=2 \mathscr{A} \operatorname{Im} c_{3}\left(\hat{\mathbf{k}} \cdot\left[\hat{\mathbf{p}}_{1} \times \hat{\mathbf{p}}_{2}\right]\right) .
$$

The term $\Delta_{c d}$ involves both the dipole-dipole and dipole-quadrupole contributions,

$$
\begin{aligned}
& \Delta_{c d}=\Delta_{c d}^{(d i p)}+\Delta_{c d}^{(q u a d r)}, \quad \text { where } \Delta_{c d}^{(d i p)}=2 \mathscr{A} \operatorname{Im}\left(f_{1} f_{2}^{*}\right)\left(\hat{\mathbf{k}} \cdot\left[\hat{\mathbf{p}}_{1} \times \hat{\mathbf{p}}_{2}\right]\right), \text { and } \\
& \Delta_{c d}^{(q u a d r)}=2 \mathscr{A} \operatorname{Im}\left[\left(f_{1} g_{s}^{*}+f_{2}^{*} g_{1}\right)\left(\hat{\mathbf{k}} \cdot \hat{\mathbf{p}}_{1}\right)-\left(f_{2} g_{s}^{*}+f_{1}^{*} g_{2}\right)\left(\hat{\mathbf{k}} \cdot \hat{\mathbf{p}}_{2}\right)\right]\left(\hat{\mathbf{k}} \cdot\left[\hat{\mathbf{p}}_{1} \times \hat{\mathbf{p}}_{2}\right]\right) .
\end{aligned}
$$



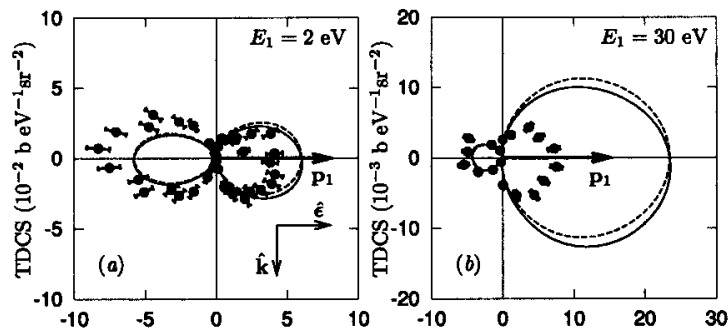

FIGURE 1. Comparison of our LOPT results for the TDCS at an excess energy of $450 \mathrm{eV}$ for the case of linear polarization with the normalized experimental data of Ref. [10]. The directions of the photon wavevector $\hat{\mathbf{k}}$ and polarization $\hat{\boldsymbol{\varepsilon}}$ are as shown in (a); the electron having energy $E_{1}$ is ejected along $\varepsilon$. Full curves: dipole-quadrupole results; dashed curves: EDA results.

Within the EDA, the CD effect is described by the term $\Delta_{c d}^{(d i p)}[3,6]$, which vanishes at equal energy sharing (because for $p_{1}=p_{2}$ one has $f_{1}=f_{2}$ ). However, the quadrupole term, $\Delta_{c d}^{(q u a d r)}$, produces a non-zero CD effect even at equal energy sharing. The dichroic term $\operatorname{Im} c_{3}\left(\hat{\mathbf{k}} \cdot\left[\hat{\mathbf{p}}_{1} \times \hat{\mathbf{p}}_{2}\right]\right)$ in Eqs. (8) and (10) results also in an unusual feature of the TDCS, a different symmetry of the TDCS with respect to two transformations: (i) $\mathbf{k} \rightarrow$ $-\mathbf{k}$ (i.e., the inversion of the photon beam direction) and (ii) $\left(\theta_{1}, \theta_{2}\right) \rightarrow\left(\pi-\theta_{1}, \pi-\theta_{2}\right)$ (i.e., the reflection of the photoelectron pair in the polarization plane) [2].

The expression for the TDCS in terms of the symmetrized amplitudes has a form identical to those in Eqs. (8)-(10) provided the following substitutions are made: $\left\{\hat{\mathbf{p}}_{1}, \hat{\mathbf{p}}_{2}, f_{1}, f_{2}, g_{1}, g_{2}, g_{s}\right\} \rightarrow\left\{\mathbf{p}_{+}, \mathbf{p}_{-}, f^{(\mathrm{g})}, f^{(\mathrm{u})}, g_{+}^{(\mathrm{g})}, g_{-}^{(\mathrm{g})}, g^{(\mathrm{u})}\right\}$. This parametrization leads to a simpler form of the TDCS for equal energy sharing [7]:

$$
\begin{aligned}
\sigma^{(e q)}= & \mathscr{A}\left\{\left[\left|f^{(\mathbf{g})}\right|^{2}+2 \operatorname{Re}\left\{f^{(\mathrm{g}) *} g_{+}^{(\mathrm{g})}\right\}\left(\hat{\mathbf{k}} \cdot \mathbf{p}_{+}\right)\right]\left|\mathbf{e} \cdot \mathbf{p}_{+}\right|^{2}+\operatorname{Re}\left\{f^{(\mathbf{g}) *} g_{-}^{(\mathrm{g})}\right\}\left(\hat{\mathbf{k}} \cdot \mathbf{p}_{-}\right)\right. \\
& \left.\times\left[2 l\left(\hat{\boldsymbol{\varepsilon}} \cdot \mathbf{p}_{+}\right)\left(\hat{\boldsymbol{\varepsilon}} \cdot \mathbf{p}_{-}\right)+(l-\mathbf{1})\left(\hat{\mathbf{k}} \cdot \mathbf{p}_{+}\right)\left(\hat{\mathbf{k}} \cdot \mathbf{p}_{-}\right)\right]\right\}+(\xi / 2) \Delta_{c d}^{(e q)} \\
\Delta_{c d}^{(e q)} \equiv & \left.\Delta_{c d}^{(q u a d r)}\right|_{p_{1}=p_{2}}=2 \mathscr{A} \operatorname{Im}\left\{f^{(\mathbf{g}) *} g_{-}^{(\mathbf{g})}\right\}\left(\hat{\mathbf{k}} \cdot\left[\mathbf{p}_{-} \times \mathbf{p}_{+}\right]\right)\left(\hat{\mathbf{k}} \cdot \mathbf{p}_{-}\right) .
\end{aligned}
$$

Numerical estimates of nondipole effects in TDCSs calculated within lowest-order perturbation theory (LOPT) in the interelectron interaction $[8,9]$ have been reported in Refs. $[2,5,7]$ for excess energies ranging from tens to hundreds of eV. In Fig. 1 we present an example of such predictions for an excess energy of $450 \mathrm{eV}$ that are compared to the experimental data of Ref. [10]. Despite the deviation of our LOPT predictions within EDA from the experimental data, one sees that both the experimental data and our nondipole results exhibit a noticeable forward-backward asymmetry as compared to our EDA results: the angular distributions of the fast electron are shifted along the direction of the vector $\mathbf{k}$, especially in the angular ranges $0<\theta_{2}<\pi / 2$ and $3 \pi / 2<\theta_{2}<2 \pi$. The relative magnitudes of such an asymmetry at particular angles $\theta_{2}$, measured by the quantity $R=\left[\sigma\left(2 \pi-\theta_{2}\right)-\sigma\left(\theta_{2}\right)\right] / \sigma\left(2 \pi-\theta_{2}\right)$, appear to be in reasonable agreement with the experimental data. Indeed, for $\theta_{1}=0.174$ (in radians), one has the theoretical value $R^{t h}=0.051$ vs. the experimentally measured value $R^{\exp }=0.049$; for $\theta_{1}=0.516$, 
$R^{\text {th }}=0.143$ vs. $R^{\text {exp }}=0.117$; for $\theta_{1}=0.868, R^{\text {th }}=0.212$ vs. $R^{\text {exp }}=0.181$; and for $\theta_{1}=1.216, R^{\text {th }}=0.229$ vs. $R^{\text {exp }}=0.398$.

\section{DIPOLE-QUADRUPOLE DDCS}

The DDCS for DPI from an initial ${ }^{1} S$ state can be written in a form that is identical to that of the angle-differential cross section for single photoionization,

$$
\frac{d^{2} \sigma}{d \Omega_{\mathbf{p}_{1}} d E_{1}}=\frac{\sigma_{0}}{4 \pi}\left\{1+\beta P_{2}\left(\left|\mathbf{e} \cdot \hat{\mathbf{p}}_{1}\right|\right)+\left[\delta+\gamma\left|\mathbf{e} \cdot \hat{\mathbf{p}}_{1}\right|^{2}\right]\left(\hat{\mathbf{k}} \cdot \hat{\mathbf{p}}_{1}\right)\right\},
$$

where, however, the singly-differential cross section (SDCS) $\sigma_{0}$, the dipole asymmetry parameter $\beta$, and the nondipole asymmetry parameters $\gamma$ and $\delta$ depend upon both the photon frequency $\omega$ and the energy sharing (i.e., upon the energy of one of the photoelectrons). For linear polarization $\left(\mathbf{e}=\mathbf{e}^{*} \equiv \hat{\varepsilon}\right), P_{2}(|\mathbf{e} \cdot \hat{\mathbf{p}}|)=P_{2}(\cos \alpha)$ is a Legendre polynomial, where $\alpha$ is the angle between the vectors $\hat{\mathbf{p}}$ and $\hat{\varepsilon}$; for circular polarization, $P_{2}(|\mathbf{e} \cdot \hat{\mathbf{p}}|)=(-1 / 2) P_{2}(\cos \phi)$, where $\phi$ is the angle between the vectors $\hat{\mathbf{p}}$ and $\hat{\mathbf{k}}$.

We have derived $a b$ initio representations for $\sigma_{0}, \beta, \gamma$, and $\delta$ at two different levels of detail. The first one is in terms of integrals of the polarization-invariant amplitudes,

$$
\begin{aligned}
& \sigma_{0}=\mathscr{A} \frac{8 \pi^{2}}{3} \int_{-1}^{1}\left[\left|f_{1}\right|^{2}+\left|f_{2}\right|^{2}+2 \operatorname{Re}\left(f_{1} f_{2}^{*}\right) x\right] d x \\
& \beta=\mathscr{A} \frac{(4 \pi)^{2}}{3 \sigma_{0}} \int_{-1}^{1}\left[\left|f_{1}\right|^{2}+\left|f_{2}\right|^{2} P_{2}(x)+2 \operatorname{Re}\left(f_{1} f_{2}^{*}\right) x\right] d x=2-\mathscr{A} \frac{8 \pi^{2}}{\sigma_{0}} \int_{-1}^{1}\left|f_{2}\right|^{2}\left(1-x^{2}\right) d x \\
& \gamma=\mathscr{A} \frac{(4 \pi)^{2}}{\sigma_{0}} \int_{-1}^{1} \operatorname{Re}\left\{f_{1}^{*}\left[g_{1}+g_{2} P_{2}(x)+2 g_{s} x\right]+f_{2}^{*}\left[g_{1} x+g_{2} P_{3}(x)+2 g_{s} P_{2}(x)\right]\right\} d x \\
& \delta=\mathscr{A} \frac{8 \pi^{2}}{\sigma_{0}} \int_{-1}^{1} \operatorname{Re}\left[f_{2}^{*}\left(g_{s}+g_{2} x\right)\right]\left(1-x^{2}\right) d x
\end{aligned}
$$

where the amplitudes $f_{1,2}(x), g_{1,2}(x)$, and $g_{s}(x)$ depend upon $x \equiv \cos \theta$. The second parametrization is in terms of an infinite sum over the reduced matrix elements,

$$
\begin{aligned}
& \sigma_{0}=(4 \pi)^{2} \frac{\mathscr{A}}{3} \sum_{l_{2}=0}^{\infty} \sum_{l_{1}=l_{2} \pm 1} \frac{\left|d_{l_{1} l_{2}}\right|^{2}}{\left(2 l_{1}+1\right)\left(2 l_{2}+1\right)}, \\
& \beta=\frac{(4 \pi)^{2}}{\sigma_{0}} \mathscr{A} \sqrt{\frac{2}{3}} \sum_{l_{2}=0}^{\infty} \sum_{l_{1}=l_{2} \pm 1} \sum_{l_{1}^{\prime}=l_{2} \pm 1} \frac{(-1)^{l_{2}}}{2 l_{2}+1} C_{l_{1} 0 l_{1}^{\prime} 0}^{20}\left\{\begin{array}{ccc}
1 & l_{1}^{\prime} & l_{2} \\
l_{1} & 1 & 2
\end{array}\right\} d_{l_{1} l_{2}} d_{l_{1}^{\prime} l_{2}}^{*}, \\
& \gamma=-\frac{(4 \pi)^{2}}{\sigma_{0}} \sqrt{10} \mathscr{A} \sum_{l_{2}=2}^{\infty} \sum_{l_{1}=l_{2} \pm 1} \sum_{l_{1}^{\prime}=l_{2}, l_{2} \pm 2} \frac{(-1)^{l_{2}}}{2 l_{2}+1} C_{l_{1} 0 l_{1}^{\prime} 0}^{30}\left\{\begin{array}{ccc}
2 & l_{1}^{\prime} & l_{2} \\
l_{1} & 1 & 3
\end{array}\right\} \operatorname{Re}\left(d_{l_{1} l_{2}} q_{l_{1}^{\prime} l_{2}}^{*}\right),
\end{aligned}
$$




$$
\begin{aligned}
\delta & =\frac{(4 \pi)^{2}}{\sigma_{0}} \frac{\mathscr{A}}{\sqrt{5}} \sum_{l_{2}=2 l_{1}=l_{2} \pm 1}^{\infty} \sum_{l_{1}^{\prime}=l_{2}, l_{2} \pm 2} \frac{(-1)^{l_{2}}}{2 l_{2}+1} \operatorname{Re}\left(d_{l_{1} l_{2}} q_{l_{1}^{\prime} l_{2}}^{*}\right) \\
& \times\left(\sqrt{3} C_{l_{1} 0 l_{1}^{\prime} 0}^{10}\left\{\begin{array}{ccc}
2 & l_{1}^{\prime} & l_{2} \\
l_{1} & 1 & 1
\end{array}\right\}+\sqrt{2} C_{l_{1} 0 l_{1}^{\prime} 0}^{30}\left\{\begin{array}{ccc}
2 & l_{1}^{\prime} & l_{2} \\
l_{1} & 1 & 3
\end{array}\right\}\right) .
\end{aligned}
$$

The dipole-quadrupole terms, which are of the order of $\omega / c$, do not appear in the SDCS given by the parameter $\sigma_{0}$ in Eqs. (15) and (16), i.e., the lowest-order nondipole corrections that contribute to the SDCS are the quadrupole-quadrupole and dipoleoctupole terms, which are of the order $(\omega / c)^{2}$ and are thus not accounted for here. Also, the parameter $\delta$ is generally non-zero. This is in contrast to SPI, for which $\delta^{S P I}$ vanishes for ionization from atomic $s$ subshells [11].

We have calculated the SDCS, $\sigma_{0}$, and the asymmetry parameters $\beta, \gamma$, and $\delta$ by the LOPT approach for an excess energy of $450 \mathrm{eV}$ for two energy sharings used in the experiment of Ref. [10]. For the angular distribution of the electron having energy $E_{1}=448 \mathrm{eV}$, we find that $\sigma_{0}=3.06 \mathrm{~b} / \mathrm{eV}, \beta=1.93, \gamma=0.51$, and $\delta=0.0043$, as compared to the CCC results $\sigma_{0}=2.54 \mathrm{~b} / \mathrm{eV}, \beta=1.92$ [10]. For the angular distribution of the electron having energy $E_{1}=420 \mathrm{eV}$, we find that $\sigma_{0}=1.19 \mathrm{~b} / \mathrm{eV}, \beta=1.83$, $\gamma=0.46$, and $\delta=0.011$, as compared to the CCC results, $\sigma_{0}=0.73 \mathrm{~b} / \mathrm{eV}, \beta=1.78$ [10].

Comparisons of the LOPT predictions for dipole-quadrupole TDCSs, as well as for the parameters $\gamma$ and $\delta$, with those calculated by a more elaborate approach would be of great interest and are now in progress [12].

\section{ACKNOWLEDGMENTS}

This work was supported in part by the U.S. Department of Energy, Office of Science, Division of Chemical Sciences, Geosciences, and Biosciences, under grant DE-FG0396ER14646, by RFBR Grant 04-02-16350, and by the joint Grant VZ-010-0 of the CRDF and the RF Ministry of Education and Sciences (NLM and AVM).

\section{REFERENCES}

1. A.I. Mikhailov, I.A. Mikhailov, A.N. Moskalev, A.V. Nefiodov, G. Plunien, and G. Soff, Phys. Rev. A 69, 032703 (2004).

2. A.Y. Istomin, N.L. Manakov, A.V. Meremianin, and A.F. Starace, Phys. Rev. A 71, 052702 (2005).

3. N.L. Manakov, S.I. Marmo, and A.V. Meremianin, J. Phys. B 29, 2711 (1996).

4. J.S. Briggs and V. Schmidt, J. Phys. B 33, R1 (2000).

5. A.Y. Istomin, N.L. Manakov, A.V. Meremianin, and A.F. Starace, Phys. Rev. Lett. 92, 063002 (2004).

6. J. Berakdar and H. Klar, Phys. Rev. Lett. 69, 1175 (1992).

7. A.Y. Istomin, N.L. Manakov, A.V. Meremianin, and A.F. Starace, Phys. Rev. A 70, 010702(R) (2004).

8. A.Y. Istomin, N.L. Manakov, and A.F. Starace, J. Phys. B 35, L543 (2002).

9. A.Y. Istomin, N.L. Manakov, and A.F. Starace, Phys. Rev. A 69, 032713 (2004).

10. A. Knapp, A. Kheifets, I. Bray, Th. Weber, A.L. Landers et al., Phys. Rev. Lett. 89, 033004 (2002).

11. J.W. Cooper, Phys. Rev. A 47, 1841 (1993).

12. A.Y. Istomin, A.F. Starace, N.L. Manakov, A.V. Meremianin, A.S. Kheifets, and I. Bray, papers are in preparation. 